\title{
P-1006
}

\section{Diet quality in obese pregnant women - could there be effects on Gestational Diabetes independent of weight gain?}

M. Neff ${ }^{1}$, R. S. Opie ${ }^{1}$

${ }^{1}$ Nutrition and Dietetics, Alfred Health, Melbourne, Australia

\section{BACKGROUND}

Obesity, excess gestational weight gain and poor diet quality during pregnancy are associated with increased risk of both maternal and fetal complications, including Gestational Diabetes Mellitus (GDM) ${ }^{1}$. Yet in Australia, standard antenatal care does not include nutritional intervention for obese pregnant women. A gap in the literature was identified with respect to intervention studies to improve diet quality and related health outcomes for obese pregnant women.

\section{AIM}

To create a Diet Quality Score to explore the degree to which changes in dietary quality occur as a result of a behavioural nutrition intervention, individually tailored for obese pregnant women in early pregnancy.

\section{METHOD}

A Diet Quality Score was devised in order to produce a single value indicative of diet quality, and hence display dietary quality changes. This score was created based on intake of food group serves, and variety and quality within each food group aligned to Australian Guide to Healthy Eating recommendations for pregnancy ${ }^{3}$. The Diet Quality Score was scored out of 100; and participants had dietary intake analysed at baseline in early pregnancy (before 20 weeks), and at 36 weeks gestation.

\section{RESULTS}

Food and fluid intake of 43 diet group participants were analysed using the Diet Quality Score at both time points.

35 women (81\%) showed Diet Quality Score improvement from baseline, with an average improvement of $34.0 \%$.

8 women (19\%) had a reduced Diet Quality Score, with an average deterioration of $5.6 \%$.
Whilst not statistically significant after adjusting for Asian ethnicity, lower rates of GDM were seen amongst obese pregnant women who received dietetic intervention when compared to the standard care control group (Opie et al $2016^{2}$ (see table below)). Excess gestational weight gain is commonly believed to be associated with incidence of $\mathrm{GDM}^{1}$. However in our study, gestational weight gain was similar for both groups, posing the question of whether diet quality improvements could be an influential factor in the lower rates of GDM development.

Table 1: Results from Obese Pregnant Women Trial, Opie R 2016

\begin{tabular}{|c|c|c|c|c|c|c|}
\hline & & $\mathbf{n}$ & $\begin{array}{c}\text { Frequency } \\
\% \text { (n) }\end{array}$ & $n$ & $\begin{array}{c}\text { Frequency } \\
\qquad \%(n)\end{array}$ & \\
\hline \multirow[t]{4}{*}{ GDM, yes } & Total Cohort & 92 & $6.5 \%(6)$ & 119 & $19.3 \%(23)$ & $*$ \\
\hline & Non-Asian & 82 & $4.9 \%(4)$ & 71 & $5.6 \%(4)$ & ns \\
\hline & Asian women & 10 & $20.0 \%(2)$ & 48 & $39.6 \%$ (19) & ns \\
\hline & & $\mathrm{n}$ & Mean (SD) & $n$ & Mean (SD) & $P^{\ddagger}$ \\
\hline $\begin{array}{c}\text { Weight gain } \\
(\mathrm{kg})\end{array}$ & Total Cohort & 82 & $10.00(4.78)$ & 52 & $9.72(5.51)$ & ns \\
\hline
\end{tabular}

A Spearman correlation was utilised to assess the strength of the relationship between the Diet Quality score and the Australian Guide to Healthy Eating. The relationship was found to be strong $(r=0.816, p$ value $<0.001)$. Further validation of the score using a food database (Foodworks $\left.{ }^{4}\right)$ supported alignment between a high Diet Quality Score and improved macro and micronutrient intake.

\section{DISCUSSION}

This dietary intervention study found that most obese pregnant women achieved improvements in diet quality, according to the Diet Quality score. Although there was no diet information available for comparison in the control group, there is evidence to suggest that overweight and obese women consume diets of poor quality during pregnancy $5,6,7$ and research has suggested that diet quality deteriorates across pregnancy ${ }^{8}$.

Whilst acknowledging that weight change during pregnancy influences outcomes, especially when excessive, we speculate that diet quality is of significant importance rather than weight status alone when considering maternal and fetal outcomes.

The exact aetiology of diet quality influence is not well understood. Poor diet quality could be linked directly with nutritional deficiencies such as folate-deficiency and neural tube defects, or nutrient excesses such as high glycaemic load and carbohydrate intake predisposing to impaired glucose tolerance and GDM. For the offspring, the nutritional environment during gestation has been thought to further influence health in adulthood through epigenetic factors ${ }^{\text {. }}$.
We were unable to capture all women for diet analysis at both time points, with $50 \%$ of women unavailable in later pregnancy. This is a limitation that prevented any further analysis regarding the relationship between GDM incidence amongst women with improvements or deteriorations in Diet Quality scores.

Furthermore, additional validation and extrapolation of the Diet Quality score to a larger cohort of obese pregnant women is necessary before explicit conclusions can be drawn. However, the positive trend to lower rates of GDM seen in the intervention group, independent of weight change, may suggest an influence from diet quality improvements seen using the Diet Quality Score. The study supports the need for wider research, whilst immediately considering the implementation of routine antenatal nutrition intervention for these high risk obese women.

\section{REFERENCES}

1. Ferrara A. Diabetes Care 2007; 30:S141 - 146.

2. Opie R.S, Neff M, Tierney A.C. Aust N Z J Obstet Gynaecol 2016; 56 (4): 364-373.

3. National Health and Medical Research Council. Healthy eating during your pregnancy

4. Foodworks 8 2012, Xyris Software, High Gate Hill, Qld, Australia.
5. Pick ME eta I. J Am Diet Assoc 2005; 105: 240-246.

6. Tsigga M et al. Public Health Nutrition 2011;14(2):290-296.

7. Hure A et al. Public Health Nutrition 2009; 12(6):853-861.

8. Moran LJ et al. Int J Obes 2013; 37(5):704-1

9. Hanley B et al. British Journal of Nutrition 2010; 104 (S1): S1-25. 